\title{
Benefícios da prática de atividade física em pessoas idosas: revisão de literatura
}

\author{
Benefits of physical activity in the elderly: a literature review \\ Beneficios de la actividad física en los ancianos: una revisión de la literatura
}

Andrea Mathes Faustino ${ }^{1 *}$, Rui Neves².

\begin{abstract}
RESUMO
Objetivo: identificar na literatura brasileira, os benefícios que a prática da atividade física pode ocasionar em pessoas idosas. Métodos: trata-se de uma revisão de literatura integrativa, de artigos publicados no período de 2014 a 2019 nas bases eletrônicas LILACS e MEDLINE, disponibilizadas pela Biblioteca Virtual em Saúde (BVS) e pela PubMed, com os seguintes descritores: Exercise (Ejercicio, Exercício); Aged (Anciano, Idoso); Motor Activity (Actividad Motora, Atividade Motora). Foram incluídos nove artigos. Resultados: Os principais benefícios referenciados nos artigos selecionados foram que a prática de atividade física favoreceu adoção de estilo de vida mais saudável, aumentou as relações sociais e rede de apoio, preservou as capacidades funcionais, reduziu a fragilidade, elevou o nível de atividade e independência entre as pessoas idosas, diminuiu o risco para o desenvolvimento de doenças ou complicações de agravos de saúde relacionados aos sistemas cardiovascular e endócrino. Considerações finais: Os estudos observados nesta revisão de literatura apontam que a prática de atividade física realizada por pessoas idosas no Brasil favorecem a prevenção e o controle das doenças crônicas não transmissíveis, aumentam a mobilidade física, ajudam a manter a capacidade funcional para as atividades de vida diária e consequentemente melhoram a qualidade de vida desta população.
\end{abstract}

Palavras-chave: Idosos, Exercício, Atividade motora.

\begin{abstract}
Objective: To identify in the Brazilian literature, the benefits that the practice of physical activity can cause in the elderly people. Methods: This is an integrative literature review, articles published from 2014 to 2019 in the electronic databases LILACS and MEDLINE, available from the Virtual Health Library (VHL) and PubMed, with the following descriptors: Exercise (Ejercicio, Exercício); Aged were included in the final sample. Results: The main benefits referenced in the selected articles were that the practice of physical activity favored the adoption of a healthier lifestyle, increased social relationships and support network, preserved functional capacities, reduced fragility, increased the level of activity and independence between women. Elderly people decreased the risk for the development of diseases or complications of health problems related to the cardiovascular and endocrine systems. Final considerations: The studies observed in this literature review point out that the practice of physical activity performed by elderly people in Brazil favors the prevention and control of chronic non-communicable diseases, increases physical mobility, helps to maintain the functional capacity for the activities of daily life and consequently improve the quality of life of this population.
\end{abstract}

Keywords: Aged, Exercise, Motor activity.

\section{RESUMEN}

Objetivo: identificar en la literatura brasileña, los beneficios que la práctica de la actividad física puede causar en los ancianos. Métodos: se trata de una revisión bibliográfica integradora de artículos publicados de 2014 a 2019 en las bases de datos electrónicas LILACS y MEDLINE, disponibles por la Biblioteca Virtual

1 Universidade de Brasília (UnB), Brasília - Distrito Federal. *E-mail: andreamathes@unb.br
${ }^{2}$ Universidade de Aveiro (UA), Aveiro - Portugal. 
en Salud (BVS) y PubMed, con los siguientes descriptores: Ejercicio (Ejercicio, Ejercicio); Anciano (Anciano, Anciano); Actividad motora (Actividad motora, Actividad motora). Se incluyeron nueve artículos. Resultados: Los principales beneficios referenciado en los artículos seleccionados fueron que la práctica de actividad física favoreció la adopción de un estilo de vida más saludable, aumentó las relaciones sociales y la red de apoyo, preservó las capacidades funcionales, redujo la fragilidad, aumentó el nivel de actividad e independencia entre las mujeres. las personas de edad avanzada, disminuyeron el riesgo de desarrollar enfermedades o complicaciones de problemas de salud relacionados con los sistemas cardiovascular y endocrino. Consideraciones finales: Los estudios observados en esta revisión de la literatura señalan que la práctica de actividad física realizada por personas mayores en Brasil favorece la prevención y el control de enfermedades crónicas no transmisibles, aumenta la movilidad física, ayuda a mantener la capacidad funcional para las actividades de vida cotidiana y en consecuencia mejorar la calidad de vida de esta población.

Palabras clave: Ancianos, Ejercicio, Actividad motora.

\section{INTRODUÇÃO}

Com o passar dos anos, o avanço da tecnologia e a automatização de certos processos tomaram conta do cotidiano e, com isso ocorreu uma redução no nível das atividades físicas ou motoras de forma geral: no trabalho, em casa e no lazer (MOURÃO ARC, et al., 2013). Diante disso, é possível verificar que o idoso que se encontra aposentado, ou seja, não se desloca para o trabalho, passa grande parte do tempo dentro de casa, ao contrário de idosos que ainda trabalham, pois precisam se deslocar de um lugar para outro pela atividade laboral. Sendo assim se faz necessário incentivar o deslocamento ativo também durante o envelhecimento (MADEIRA MC, et al., 2013).

A prática regular de atividade física tem papel fundamental na prevenção e controle das doenças crônicas não transmissíveis, favorecendo melhor mobilidade, capacidade funcional e qualidade de vida, em todas as fases da vida, principalmente quando se trata do processo de envelhecimento. Além de estimular a prática de exercícios físicos entre pessoas idosas também é crucial incluir a estas atividades, o fortalecimento muscular e exercícios de equilíbrio a fim de melhorar a aptidão física e a manutenção das capacidades funcionais, e consequentemente maior independência física para esta população (MATSUDO SMM, 2009).

A inclusão da prática de Atividade Física (AF) regular, mesmo aquelas não estruturadas, ou seja, aquelas atividades relacionadas ao cotidiano de um indivíduo, como atividades de lazer, tarefas domésticas, oferecem benefícios em detrimento de uma rotina sedentária, e assim são importantes para reduzir e até mesmo reverter declínios de ordem física, psíquica e social, psicológico e social. Condições que podem estar associadas ao processo de envelhecimento não ativo. Mesmo sabendo dos benefícios da prática de $\mathrm{AF}$, pouco ainda é incentivado pelos profissionais da saúde, e assim a taxa de inatividade entre a população acima dos 65 anos, chega a ser de 32,3\% nesta população (SOUZA JL, et al., 2014).

A Organização Mundial da Saúde (OMS) descreve a inatividade física como o quarto fator de risco global para a mortalidade, por ser um dos principais fatores que influenciam para o desenvolvimento de doenças crônicas não transmissíveis no Brasil. Estudos recentes relatam a prática de AF regular como sendo capaz de retardar o desenvolvimento e progresso de doenças crônicas e preservar o funcionamento físico e assim reduzir o processo fisiológico do envelhecimento (SILVA LJ, et al., 2012; REZENDE LF, et al., 2015; ZAGO AS, 2010).

Em muitos países, e especialmente nos países em desenvolvimento, algumas estratégias para auxiliar pessoas mais velhas a se manterem saudáveis e ativas por mais tempo são uma necessidade iminente. $O$ conceito de envelhecimento ativo vai além das relações com a força física, mas vai direto a participação social do idoso, apontando para uma nova concepção sobre a velhice, com maior engajamento social. Nessa proposta, as pessoas mais velhas passam a ser vistas, para além de aspectos meramente orgânicos, podendo ser qualificadas como contribuintes e beneficiárias do desenvolvimento social. Conceitualmente 0 envelhecimento ativo se apoia em três pilares: saúde, segurança e participação (MASSI G, et al., 2018). 
A falta de AF entre pessoas idosas desencadeiam agravos de saúde e podem levar a dependência e declínio funcional. As alterações físicas podem estar associadas aos sistemas cardiovasculares e musculoesquelético, como por exemplo a diminuição da força, flexibilidade e equilíbrio, aumento da resistência cardiovascular (SOUZA JL, et al., 2014; VIEIRA SCAL, et al., 2015).

Algumas características podem influenciar para um comportamento não propício a prática de $\mathrm{AF}$, como por exemplo: possuir oitenta anos ou mais; ser do sexo feminino; apresentar capacidade funcional reduzida e já ter sofrido quedas (FERREIRA OGL, et al., 2010).

O sedentarismo também pode estar relacionado a idosos com baixa renda sendo que idosos que recebem entre um a cinco salários mínimos são menos ativos em relação a idosos com renda superior a 5,1 salários, o que demonstra que a prática regular de AF pode ser uma questão de oportunidade de acesso e de aspectos de capacidade funcional preservada (SONATI JG, et al., 2011).

Aderir a um comportamento fisicamente ativo, e conseguir manter-se neste novo estilo de vida é algo considerado complexo e dinâmico, além de ser determinado por múltiplos fatores como os de ordem pessoal no que tange a motivação individual e comportamental, e por questões ambientais que podem estimular ou não a praticar atividades físicas ao ar livre ou em ambientes fechados (BARROS MB, IAOCHITE RT, 2012).

Diminuir o sedentarismo e promover estilos de vida mais saudáveis com a participação da atenção básica à saúde e de seus profissionais pode representar um grande impacto na melhoria dos índices de saúde populacional e nos custos relacionados à gestão dos serviços (SONATI JG, et al., 2011).

As práticas de AF a fim de favorecer uma vida mais saudável, devem ser metas tanto para idosos que estejam na comunidade tanto os institucionalizados. Os efeitos do sedentarismo em idosos institucionalizados podem ser ainda maiores, podendo ser determinantes para o comprometimento no desempenho das atividades básicas de vida diária, aumento do número de quedas e elevado número de complicações relacionadas às doenças crônicas não transmissíveis (SOUZA JL, et al., 2014).

Muitas iniciativas para aumentar o nível da AF entre a população idosa no Brasil já foram criadas, a fim de preservar a saúde física e mental como um dos direitos assegurados pelo Estatuto do Idoso, lei no 10741. Contudo a implementação de programas como políticas públicas ainda são um desafio para os gestores públicos, assim adotar um estilo de vida ativo ainda é um avanço necessário para se tornar uma iniciativa de promoção a saúde junto a idosos brasileiros (SOUZA JL, et al., 2014).

A Lei número 8842/949 e o Decreto № 1948/96da Política Nacional do Idoso asseguram o direito à pessoa idosa e recomendam a criação de condições para a promoção de autonomia, integração e participação efetiva na sociedade, reafirmando o direito à saúde nos diversos níveis de atendimento. Desta forma, os profissionais da área de saúde têm o dever ético, moral e cívico de adotar atitudes que privilegiem o idoso (ZAGO AS, 2010; SILVA MRF, YAZBEK MC, 2014).

Assim este trabalho teve como objetivo identificar na literatura brasileira, os impactos e benefícios que a prática da AF pode ocasionar entre pessoas idosas. Sabendo da importância que o envelhecimento ativo tem em relação a saúde e bem-estar geral, elegemos como prioridade verificar se existem atualizações sobre os efeitos da inclusão de exercícios físicos, e como podem ser incorporadas no cotidiano de idosos, além de sintetizar evidências na literatura sobre investigações no Brasil.

\section{MÉTODOS}

Trata-se de um estudo do tipo revisão de literatura integrativa, cuja pergunta norteadora elaborada pelos pesquisadores foi $\ll$ Quais os benefícios que a prática da atividade física pode ocasionar entre as pessoas idosas? >>. A fim de se cumprir as etapas de um estudo de revisão de literatura, já descritos em outras pesquisas, optou-se por seguir a sistematização descrita a seguir: estabelecer uma hipótese e objetivos acerca da revisão integrativa; determinar os critérios de inclusão e/ou exclusão de artigos para se obter a seleção da amostra final; definir quais seriam as informações que responderão a pergunta norteadora e 
organizar por meio de categoriais a serem apresentadas como resultados e discussão. A etapa final é a apresentação da revisão em si, com todos os elementos identificados após as leituras e organização dos subtemas principais verificados durante o processo (MENDES KDS, et al., 2008).

O levantamento de dados bibliográficos ocorreu nos meses de dezembro de 2019 e janeiro de 2020, a partir de artigos publicados no período dos anos de 2014 a 2019 nas seguintes bases eletrônicas: Medical Literature Analysis and Retrieval Sistem on-line (MEDLINE), e Literatura Latino-Americana e do Caribe em Ciências da Saúde (LILACS) disponibilizada pela Biblioteca Virtual em Saúde (BVS). Os filtros utilizados para as buscas foram: texto completo (disponível), base de dados (MEDLINE e LILACS), limite (humanos e idoso), País / Região como assunto (Brasil), Idioma (inglês, português e espanhol), país de afiliação (Brasil).

Para a realização das buscas nas bases de dados foram utilizados os seguintes descritores de assunto em saúde (DECS/MESH): Exercise (inglês), Ejercicio (espanhol), Exercício (português), Aged (inglês), Anciano (espanhol), Idoso (português), Motor Activity (inglês), Actividad Motora (espanhol), Atividade Motora (português) (Quadro 1).

Quadro 1 - Estratégia de busca. Brasília - DF, 2019.

\begin{tabular}{|c|l|}
\hline & Exercício (DECS), Idoso (DECS), Atividade Motora (DECS) \\
\hline OR & Exercise (Mesh), Aged (Mesh), Motor Activity (Mesh) \\
\hline OR & Ejercicio (DECS), Anciano (DECS) Actividad Motora (DECS) \\
\hline AND & (collection:("06-national/BR" OR "05-specialized") OR db:("LILACS" OR "MEDLINE") \\
\hline
\end{tabular}

Fonte: Faustino AM, Neves R, 2020.

Assim quanto aos critérios de inclusão dos artigos selecionados para a presente revisão, foram observadas as seguintes condições: ser artigo publicado nos idiomas português, inglês ou espanhol; possuir resumos disponíveis nas bases de dados para a leitura e posterior seleção; estar disponível na íntegra com acesso on-line nas bases de dados pesquisadas; e estar publicados no período compreendido entre os anos de 2014 a 2019, cuja temática principal abordasse os benefícios da AF em pessoas idosas, em estudos desenvolvidos no Brasil.

As publicações selecionadas foram lidas integralmente e posteriormente, categorizadas por tipo e objetivo do estudo, por amostra estudada, síntese de conclusões, instrumentos utilizados, entre outras variáveis observadas e registradas em instrumento próprio para a coleta de dados que foi elaborado pelos autores. A fim de sistematizar as informações de interesse dos artigos incluídos, os dados foram organizados no modelo descritivo em tabelas previamente elaboradas, o que possibilitou a identificação e classificação nas categorias de análise.

\section{RESULTADOS}

Foram encontrados 65 artigos nas bases de dados MEDLINE e LILACS disponibilizadas pela BVS e pelo PubMed, porém, após a leitura dos resumos, somente onze publicações atenderam aos critérios de inclusão (Figura 1).

Quanto ao ano de publicação, os onze artigos foram publicados entre os anos de 2014 a 2019, indicando que não há muitas pesquisas atuais relacionadas ao tema, o que dificulta a comparação dos estudos, por serem de delineamentos distintos com populações não uniformes (Figura 1). 
Figura 1 - Diagrama da seleção de estudos que fizeram parte da amostra.

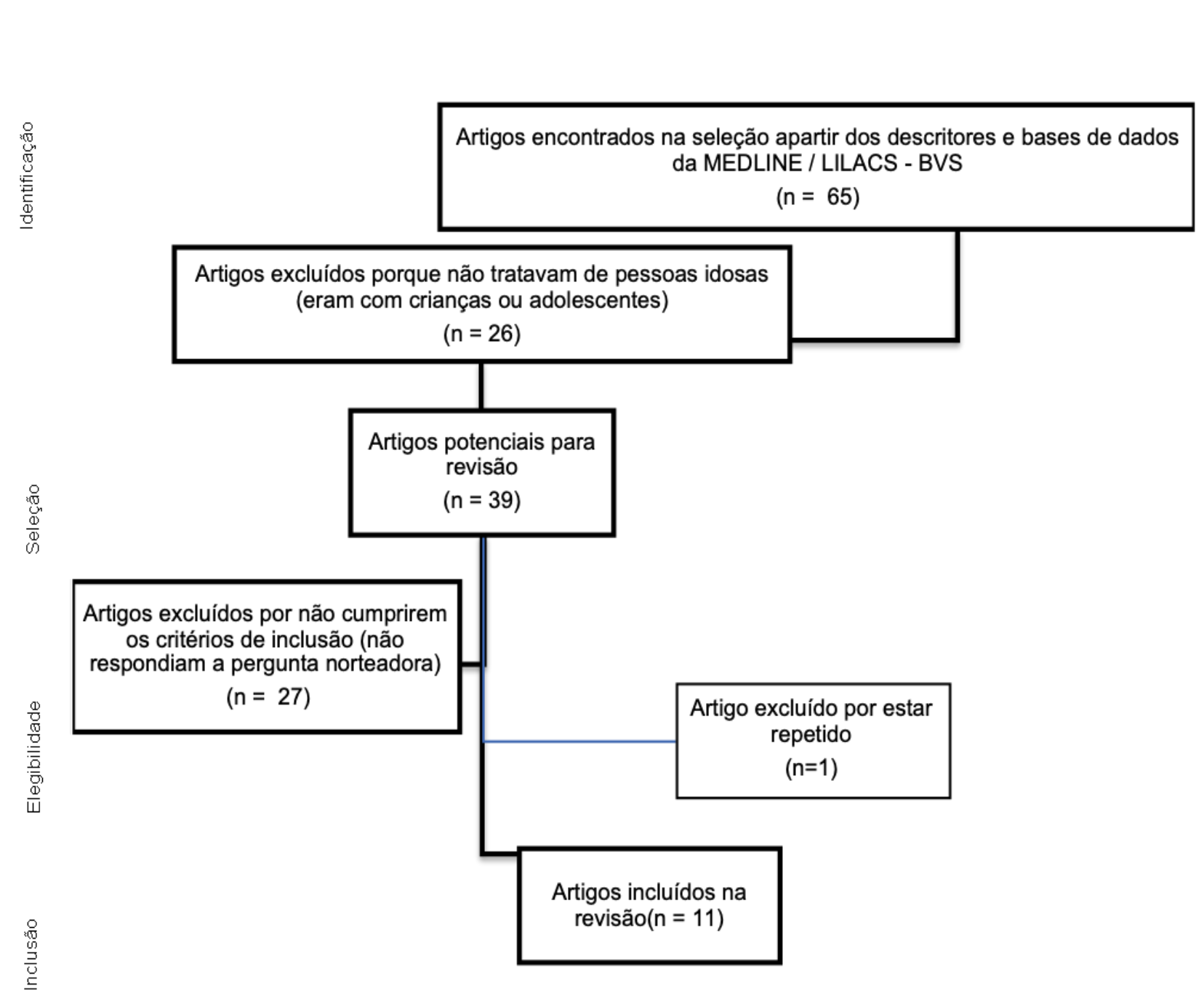

Fonte: Faustino AM, Neves R, 2020.

Quanto ao tipo de estudo, seis deles tinham o delineamento de corte transversal, com abordagens descritivas e quantitativas em sua maioria. Em relação a região de origem e desenvolvimento dos estudos seis estudos eram da região Sul do país e três da região sudeste. O que mostra pouca representatividade de amostras com idosos nas regiões norte, nordeste e centro oeste do Brasil. Não foram identificados nas bases de dados pesquisadas artigos publicados no ano de 2018. Quanto ao tipo de periódico cinco das publicações incluídas estavam em revistas da área da Saúde Pública e da Saúde Coletiva (Quadro 2).

Em relação aos benefícios da AF na vida dos idosos que participaram dos estudos evidenciaram que a adoção de estilo de vida mais saudável entre idosos praticantes de AF, pode aumentar as relações sociais e rede de apoio, promoção da saúde e diminuição do impacto das doenças crônicas. Além de reduzir fragilidades, manter as capacidades funcionais e cognitivas o que permitiria um envelhecimento mais ativo e pessoas idosas mais independentes e com autonomia (Quadro 2).

Não foram observadas diferenças quando comparados com adultos mais jovens quanto a duração e a frequência da $A F$, sendo o mais importante a prática dela. Em relação ao tamanho da amostra incluída nos estudos avaliados esta variou de acordo com a abordagem e tipo de estudo (Quadro 2). 


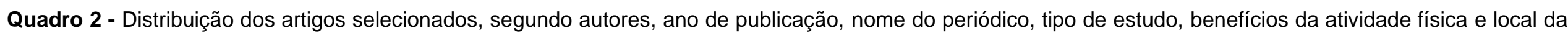
pesquisa no Brasil $(n=11)$.

\begin{tabular}{|c|c|c|c|c|c|}
\hline Autores & Ano publicação & Nome do periódico & Tipo de estudo & Benefícios da Atividade Física & $\begin{array}{c}\text { Local da Pesquisa no } \\
\text { Brasil }\end{array}$ \\
\hline $\begin{array}{l}\text { Freitas CS, } \\
\text { et al. }\end{array}$ & 2014 & Rev. Kairós & $\begin{array}{l}\text { Estudo descritivo com } \\
\text { abordagem quantitativa }\end{array}$ & $\begin{array}{l}\text { A relação de idosos em grupos de } \\
\text { convivência realizando AF do tipo } \\
\text { caminhada ou ginástica, pelo menos duas } \\
\text { vezes na semana, pode contribuir para } \\
\text { que eles sejam mais ativos e } \\
\text { independentes. }\end{array}$ & Rio Grande do Sul \\
\hline $\begin{array}{c}\text { Cabral } \\
\text { ACA, et al. }\end{array}$ & 2014 & $\begin{array}{l}\text { Rev. pesqui. cuid. } \\
\text { fundam. }\end{array}$ & $\begin{array}{l}\text { Pesquisa quantitativa } \\
\text { pré-experimental }\end{array}$ & $\begin{array}{l}\text { O programa de treinamento de resistência } \\
\text { mostrou efeitos positivos na melhoria da } \\
\text { capacidade funcional dos idosos. O } \\
\text { programa afetou a composição corporal. }\end{array}$ & Pará \\
\hline $\begin{array}{l}\text { Del Duca } \\
\text { GF, et al. }\end{array}$ & 2014 & $\begin{array}{l}\text { Ciência \& Saúde } \\
\text { Coletiva }\end{array}$ & $\begin{array}{l}\text { Estudo transversal de } \\
\text { base populacional }\end{array}$ & $\begin{array}{l}\text { As AF mais intensas praticadas no lazer } \\
\text { também foram mais comuns entre os } \\
\text { adultos mais jovens. Entretanto, não foram } \\
\text { constatadas diferenças significativas entre } \\
\text { as idades quando observadas a duração e } \\
\text { a frequência semanal de prática de AF } \\
\text { nesse domínio. }\end{array}$ & Santa Catarina \\
\hline $\begin{array}{l}\text { Fernandes } \\
\mathrm{AP} \text {, et al. }\end{array}$ & 2015 & Cad. Saúde Pública & Inquérito Domiciliar & $\begin{array}{l}\text { A utilização pela população residente entre } \\
\text { ela idosos dos "Programas Academias da } \\
\text { Cidade" podem favorecer para a equidade } \\
\text { nos benefícios do acesso à AF e assim } \\
\text { promover saúde entre esta população. }\end{array}$ & Minas Gerais \\
\hline $\begin{array}{l}\text { Lopes DC, } \\
\quad \text { et al. }\end{array}$ & 2015 & $\begin{array}{l}\text { Estud. interdiscip. } \\
\text { Envelhec. }\end{array}$ & $\begin{array}{l}\text { Estudo descritivo, } \\
\text { populacional, de base } \\
\text { domiciliar, de corte } \\
\text { transversal }\end{array}$ & $\begin{array}{l}\text { Programas de AF propostos para idosos } \\
\text { devem levar em conta não somente os } \\
\text { benefícios cardiovasculares, mas também } \\
\text { a preservação das capacidades funcionais } \\
\text { dessa população. }\end{array}$ & Rio Grande do Sul \\
\hline Marques & 2016 & Cad. Saúde Pública & Estudo transversal, & Aumento das relações sociais e rede de & Santa Catarina \\
\hline
\end{tabular}

REAS/EJCH | Vol.12(5) | e3012 | DOI: https://doi.org/10.25248/reas.e3012.2020 Página 6 de 10 
Revista Eletrônica Acervo Saúde / Electronic Journal Collection Health | ISSN 2178-2091

\begin{tabular}{|c|c|c|c|c|c|}
\hline Autores & Ano publicação & Nome do periódico & Tipo de estudo & Benefícios da Atividade Física & $\begin{array}{l}\text { Local da Pesquisa no } \\
\text { Brasil }\end{array}$ \\
\hline LP, et al. & & & $\begin{array}{l}\text { longitudinal, } \\
\text { populacional } \\
\text { domiciliar }\end{array}$ & apoio entre idosos praticantes de AF. & \\
\hline $\begin{array}{l}\text { Turi BC, et } \\
\text { al }\end{array}$ & 2016 & $\begin{array}{l}\text { Ciência \& Saúde } \\
\text { Coletiva }\end{array}$ & Estudo transversal & $\begin{array}{l}\text { A prática de exercício pode conferir } \\
\text { benefícios tangíveis para a prevenção e } \\
\text { reversão de fatores de risco para } \\
\text { Síndrome Metabólica entre adultos e } \\
\text { idosos. }\end{array}$ & São Paulo \\
\hline $\begin{array}{l}\text { Nascimento } \\
\mathrm{M} \text {, et al. }\end{array}$ & 2016 & $\begin{array}{l}\text { Rev. bras. ativ. fís. } \\
\text { saúde }\end{array}$ & Relato de experiência & $\begin{array}{l}\text { A participação em programas de AF } \\
\text { contribuíram diretamente para que uma } \\
\text { parcela da população local possa } \\
\text { envelhecer com vitalidade, o que, por sua } \\
\text { vez, reduzirá a fragilidade nesse grupo. }\end{array}$ & Pernambuco e Bahia \\
\hline $\begin{array}{l}\text { Blay SL, et } \\
\quad \text { al }\end{array}$ & 2016 & $\begin{array}{l}\text { Journal American of } \\
\text { Geriatric }\end{array}$ & Estudo Transversal & $\begin{array}{l}\text { A inserção da prática de atividade em } \\
\text { idosos com depressão podem reduzir as } \\
\text { complicações associadas a esta } \\
\text { comorbidade, bem como melhorar as } \\
\text { condições de saúde mental, sendo mesmo } \\
\text { atividade física leves ou moderadas. }\end{array}$ & Rio Grande do Sul \\
\hline $\begin{array}{l}\text { Ferrari TK, } \\
\text { et al. }\end{array}$ & 2017 & Cad. Saúde Pública & $\begin{array}{l}\text { Estudo transversal de } \\
\text { base populacional }\end{array}$ & $\begin{array}{l}\text { Adoção de estilo de vida mais saudável } \\
\text { entre idosos praticantes de AF. }\end{array}$ & São Paulo \\
\hline $\begin{array}{l}\text { Dumith SC, } \\
\text { et al }\end{array}$ & 2019 & $\begin{array}{l}\text { Rev. Bras. } \\
\text { Epidemiologia }\end{array}$ & Estudo Transversal & $\begin{array}{l}\text { Os benefícios observados quanto a prática } \\
\text { de atividade física moderada, foi que esta } \\
\text { pode conferir maior proteção aos } \\
\text { praticantes, no caso adultos e idosos em } \\
\text { relação ao estresse, e quanto a prática de } \\
\text { atividade física vigorosa foi fator de } \\
\text { proteção contra a diabetes, hipertensão e } \\
\text { obesidade. }\end{array}$ & Rio Grande do Sul \\
\hline
\end{tabular}

Fonte: Faustino AM, Neves R, 2020.

REAS/EJCH | Vol.12(5) | e3012 | DOI: https://doi.org/10.25248/reas.e3012.2020 Página 7 de 10 


\section{DISCUSSÃO}

A partir das análises dos conteúdos e dos resultados obtidos durante as buscas, foram identificadas duas categorias principais, as quais foram agrupadas em dois subtemas:"Estilo de vida saudável e rede de apoio social favorecendo a prática da atividade física"e "Melhoria da capacidade física e cognitiva associada a prática de atividade física", que a seguir estão apresentados.

\section{Estilo de vida saudável e rede de apoio social favorecendo a prática da atividade física}

Nos estudos incluídos na presente revisão, a prevalência de estilo de vida saudável foi maior entre idosos, seguida pelos adultos e adolescentes. O consumo alimentar foi o principal responsável pelo estilo de vida não saudável entre as pessoas mais jovens, destacando assim a importância de intervenções para a promoção do estilo de vida saudável e, principalmente, da dieta adequada durante todo processo de envelhecimento. Sabe-se que as teorias de estilo de vida saudável recomendam que a saúde de uma população seja orientada pelos determinantes sociais, culturais, econômicos, históricos e políticos. Só por meio de mudanças significativas que alterem estes fatores é que podem assim favorecer a manutenção de estilos de vida mais saudável durante toda a vida, em consonância com políticas públicas brasileiras, já existentes, contudo, ainda não efetivas (FERRARI TK et al., 2017).

Algumas mudanças nas relações sociais tiveram um efeito positivo sobre a qualidade de vida, e os resultados reafirmaram a importância da AF para o envelhecimento saudável, uma vez que a procura da vida ativa foi mais importante do que permanecer inativo. Além disto, alguns dos fatores que podem contribuir para a qualidade de vida entre idosos foi o incentivo a participação em atividades sociais, voltar ao trabalho, usar a Internet e praticar AF. Também foi observado em grupo de idosos que continuar vivendo com familiares teve um efeito negativo sobre qualidade de vida e consequentemente na capacidade funcional (MARQUES LP, et al., 2016). Fato este que pode ser explicado pela atitude de muitos familiares superprotegerem os idosos e assim diminuir sua autonomia, pois por não conhecerem o processo de envelhecimento podem considerar que todo idoso é frágil e assim realizar por eles algumas atividades que eles próprios poderiam estar fazendo, o que reduz sua atividade e independência física e funcional.

Os benefícios da prática da AF estão diretamente relacionados ao acesso a programas abertos a população que incluam atividades físicas e maximizem as relações interpessoais e intergeracionais. Os resultados de alguns estudos sugerem a potencialidade de programas abertos a comunidades em influenciar a prática de AF no lazer da população residente mais próxima, sendo, portanto, uma ação estratégica para mitigar as iniquidades em relação às práticas da $A F$, ou seja, tornando algo mais acessível e intrínseco as atividades da comunidade (FERNANDES AP, et al., 2015).

A incorporação de programas que ofereçam AF a idosos, com base nos relatos dos idosos participantes, foi essencial para contribuir diretamente para que uma parte da população local possa envelhecer com vitalidade, o que por sua vez, a adoção destas práticas nas rotinas de vida diária poderá reduzir o desenvolvimento de fragilidades associadas ao envelhecimento (NASCIMENTO M, et al., 2016).

O tipo de atividade esteve associado ao nível de escolaridade e renda do individuo, ou seja, atividades como musculação esteve relacionada a pessoas com maior renda, por exigirem espaços, muitas vezes privados, que dificultam o acesso ao público em geral. Para os idosos alguns tipos de atividades podem não fazer parte do rol de prática, pelo fato de que não conseguem ter acesso pela questão das dificuldades financeiras que permeiam as baixas rendas de idosos no Brasil. Contudo, não foram observadas diferenças significativas entre as faixas etária quando observadas a duração da atividade e a frequência semanal de prática nesse domínio (DEL DUCA GF, et al., 2014).

\section{Melhoria da capacidade física e cognitiva associada a prática de atividade física}

Ao se tratar da melhora dos fatores de risco para as doenças crônicas não transmissíveis, alguns estudos observaram que quanto menor o engajamento com a prática de atividades físicas em diferentes domínios há uma maior chance da prevalência de fatores de risco para a síndrome metabólica, e que idosos com menores níveis de AF no lazer tiveram maiores chances de ocorrência de doenças como o diabetes 
mellitus, hipercolesterolemia e a síndrome metabólica. Também foi possível observar melhora nas medidas de composição corporal e autonomia funcional dos idosos que praticaram atividade física (CABRAL ACA, ET AL., 2014; TURI BC et al., 2016). Achados de estudos sugerem que somente altos níveis de AF são associados a atividades básicas e funcionais preservadas. A AF apresenta-se eficiente na promoção da saúde durante o processo de envelhecimento, com a manutenção da saúde e melhor qualidade de vida (LOPES DC, et al., 2015;FREITAS CS, et al., 2014).

Alguns tipos de treinamento físico podem favorecer alguns quesitos com efeito positivo para a capacidade funcional e composição corporal de mulheres idosas. Ações de promoção da AF entre a população idosa devem ser previamente planejadas levando em consideração os distintos interesses populacionais e individuais para o estímulo à sua prática no lazer. A prática de AF nas mais diferentes intensidades, mesmo aquelas praticadas como lazer, como ginástica em geral ou futebol, foram as mais comuns entre adultos (DEL DUCA GF, et al, 2014).

Em uma população de adultos e idosos, quando foram analisadas as condições de saúde, verificou-se que praticar atividade física moderada, ou seja, aquelas atividades que exigem esforço físico médio e assim quem as pratica, tende a respirar um pouco mais rápido do que o normal, sendo os exemplos nadar, pedalar e praticar esportes por lazer ou diversão, podem ter efeito protetor contra doenças crônicas como obesidade, hipertensão e diabetes. Além disto a prática de atividade física moderada, também conferiu efeito protetor para o estresse, o que traz benefícios para a saúde mental (DUMITH SC, et al, 2019).

Associar a AF ao tratamento de depressão em idosos, mostrou-se como terapia complementar com resultados benéficos em comparação com a população sedentária. Foi observado redução nos níveis de complicações associadas a depressão, pois trata-se de uma terapia com baixo índices de efeitos colaterais em relação a outras terapêuticas incluindo as farmacológicas. Assim do ponto de vista da saúde pública, favorecer e disponibilizar recursos e espaços seguros para a realização de atividade física entre idosos com depressão podem reduzir custos associados a maiores complicações durante o tratamento para a depressão (BLAY SL, et al, 2016).

Esta revisão de literatura traz como limitação a busca por artigos dos últimos cinco anos, visto que os temas relacionados a AF em pessoas idosas são discutidos nos periódicos nacionais e internacionais anteriores a este período. Contudo por meio dos artigos selecionados reuniu experiências e evidências científicas que poderão subsidiar as equipes de saúde, bem como gestores públicos na elaboração e implementação de programas de atividade física que possam beneficiar os idosos no Brasil.

\section{CONSIDERAÇÕES FINAIS}

Os estudos observados nesta revisão de literatura apontam que a prática de AF por pessoas idosas no Brasil favorecem a prevenção e o controle das doenças crônicas não transmissíveis, aumentam a mobilidade física, ajudam a manter a capacidade funcional e cognitiva para as atividades de vida diária e consequentemente melhoram a qualidade de vida desta população. Os benefícios da AF entre pessoas idosas são influenciados pela motivação individual e também por questões culturais, econômicas e políticas. Eles influenciam diversos níveis, desde o aumento das relações sociais e expansão da rede de apoio até a promoção da saúde, diminuindo o impacto das doenças crônicas. Estimular e garantir o acesso às práticas de AF ainda é um grande desafio no Brasil, pois apesar de existirem muitas políticas públicas que favorecem estas ações, faltam incentivos para a execução e manutenção delas. Ainda existem muitas lacunas de ordem social e econômica que precisam ser asseguradas a esta população, e isto perpassa aos interesses do poder público e privado.

\section{REFERÊNCIAS}

1. BARROS MB, IAOCHITE RT. Autoeficácia para a prática de atividade física por indivíduos adultos. Motriz. $2012 ; 8(2), 32-41$. 
2. BLAY SL, et al. Is Higher Frequency of Physical Activity Associated with Reduced Prevalence of Depression Among Older Community Residents? A Study from Brazil. J Am Geriatr Soc. 2016 set; 64 (9): e42-e44.

3. CABRAL ACA, et al. Body composition and functional autonomy of older adult women after a resistance training program. Revista de Pesquisa: Cuidado é Fundamental Online. 2014; 6(1),74-85.

4. DEL DUCA GF, et al. Atividades físicas no lazer entre adultos de Florianópolis, Santa Catarina, Brasil: estudo populacional sobre as características das práticas e de seus praticantes. Ciênc. saúde coletiva. 2014; 19(11), 45954604.

5. DUMITH SC, et al. Preditores e condições de saúde associados à prática de atividade física moderada e vigorosa em adultos e idosos no sul do Brasil. RevBrasEpidemiol, 2019; 22: E190023.

6. FERNANDES AP, et al. Atividade física de lazer no território das Academias da Cidade, Belo Horizonte, Minas Gerais, Brasil: o efeito da presença de um programa de promoção da saúde na comunidade. Cad. Saúde Pública. 2015; 31( Suppl 1 ), 195-207.

7. FERRARI TK et al. Estilo de vida saudável em São Paulo, Brasil. Cadernos de Saúde Pública. 2017; 33(1), e00188015.

8. FERREIRA, OGL et al. O envelhecimento ativo sob o olhar de idosos funcionalmente independentes. Rev. esc. enferm. USP, São Paulo. 2010; 44(4), 1065-1069.

9. FREITAS CS, et al. Qualidade de vida de idosos ativos e insuficientemente ativos do município de Santa Maria (RS). Revista Kairós: Gerontologia. 2014; 17(1): 57-68.

10. LOPES DC, et al. Níveis de atividade física relacionados às atividades básicas e funcionais em idosos do Rio Grande do Sul - Brasil / Levelsofphysicalactivityrelatedtodaily living andfunctionalactivities in olderadultsfrom Rio Grande do Sul - Brasil. Estud. interdiscip. envelhec. 2015; 20(1): 73-85.

11. MADEIRA MC, et al. Atividade física no deslocamento em adultos e idosos do Brasil: prevalências e fatores associados. Cad. Saúde Pública. 2013; 29(1):165-174.

12. MARQUES LP, et al. Quality of life and its association with work, the Internet, participation in groups and physical activity among the elderly from the EpiFloripa survey, Florianópolis, Santa Catarina State, Brazil. Cad. SaúdePública. 2016; 32 (12): e00143615.

13. MASSI G, et al. Envelhecimento ativo: um relato de pesquisa-intervenção. Rev. CEFAC. $2018 ; 20$ (1): 5-12.

14. MATSUDO SMM. Envelhecimento, atividade física e saúde. BIS, Bol. Inst. Saúde. São Paulo. 2009; (47), 76-79.

15. MENDES KDS, et al. Revisão integrativa da Literatura: método de pesquisa para a incorporação de evidências na saúde e na enfermagem. Texto contexto Enfermagem, Florianópolis. 2008; 17: 758-764.

16. MOURÃO ARC, et al. Atividade física de idosos relacionada ao transporte e lazer, Maceió, Brasil. Rev. Saúde Pública. 2013; 47 (6): 1112-1122.

17. NASCIMENTO M, et al. Programa Vida Ativa: esporte e lazer promoção da saúde e qualidade de vida do idoso, em Petrolina-PE e Juazeiro-BA. Rev. bras. ativ. fís. saúde. 2016; 21(6): 593-599.

18. REZENDE LF, et al. Effect of Physical Inactivity on Major Noncommunicable Diseases and Life Expectancy in Brazil. J PhysAct Health. 2015; 12(3):299-306.

19. SILVA LJ, et al. Association between levels of physical activity and use of medication among older women. Cad. SaúdePública. 2012; 28 (3): 463-471.

20. SILVA MRF, YAZBEK MC. Proteção social aos idosos: concepções, diretrizes e reconhecimento de direitos na América Latina e no Brasil. Revista Katálysis. 2014; 17(1), 102-110.

21. SONATI JG, et al. Body weight as an indicator of fat-free mass in active elderly women. Maturitas. 2011;68(4):37881.

22. SOUZA JL, et al. El efecto de lainactividad física enlaaptitud física y funcional en personas mayores institucionalizados de São Caetano do Sul, São Paulo, Brasil. Revista Ciencias de laActividad Física. 2014; 15(2): 63-72.

23. TURI BC et al. Low levels of physical activity and metabolic syndrome: cross-sectional study in the Brazilian public health system. Ciência \& Saúde Coletiva. 2016; 21(4): 1043-1050.

24. VIEIRA SCAL, et al. A força muscular associada ao processo de envelhecimento. Ciências Biológicas e da Saúde. $2015 ; 3(1): 93-102$.

25. ZAGO AS. Exercício físico e o processo saúde-doença no envelhecimento. Rev. bras. geriatr. gerontol. $2010 ; 13$ (1): 153-158. 